\title{
On the Use of a Suppression Filter for CDMA Overlay
}

\author{
Jiangzhou Wang, Senior Member, IEEE
}

\begin{abstract}
This paper is concerned with a direct-sequence codedivision multiple-access (DS-CDMA) system operating over a Rayleigh fading and sharing common spectrum with a narrowband waveform. A suppression filter at a receiver is employed to reduce the narrow-band interference. We evaluate the average up-link bit error rate (BER) performance and investigate how the performance is influenced by various parameters, such as the number of taps of the suppression filter, the number of multipleaccess users, the ratio of narrow-band interference bandwidth to the spread-spectrum bandwidth, the interference power to signal power ratio, the ratio of the offset of the interference carrier frequency from the spread-spectrum carrier frequency to the half spread-spectrum signal bandwidth, and so on.
\end{abstract}

Index Terms-CDMA, fading channels, narrow-band interference suppression.

\section{INTRODUCTION}

D UE TO THEIR well-known multiple-access capability, antimultipath capability, and antiinterference capability, spread-spectrum techniques have widely been studied and applied. For example, [1]-[7] study the bit error rate (BER) performance of code-division multiple-access (CDMA) systems over either additive white Gaussian noise (AWGN) channels or fading channels. CDMA allows many users to access the channel simultaneously, similar to time-division multiple access (TDMA) or frequency-division multiple access (FDMA), but it does not need severe system synchronization like TDMA or severe frequency assignments like FDMA. Moreover, CDMA is more effective in combating multipath and narrow-band interference than either TDMA or FDMA. Alternately, [8]-[13] present performance analysis of spread-spectrum systems employing suppression filters against narrow-band interference over AWGN channels. The suppression filter is very effective in reducing strong narrow-band interference. In particular, a Wiener-type filter, described in many of the papers, employs a tapped delay line structure to first predict and then subtract out the narrow-band interference.

Reference [14] has proposed allowing a CDMA network to be overlaid on top of existing microwave narrow-band users that occupy a part of the spread-spectrum system bandwidth (BW). In fact, this is another advantage of CDMA over either TDMA or FDMA, because this will increase the overall

Manuscript received August 12, 1996; revised July 13, 1998. This work was supported by the Research Grant Council (RGC) of the Hong Kong Government.

The author is with the Department of Electrical and Electronic Engineering, University of Hong Kong, Hong Kong (e-mail: jwang@eee.hku.hk).

Publisher Item Identifier S 0018-9545(99)01071-3. spectrum capability. However, such an application must be considered carefully, because the CDMA (wide-band) users and the narrow-band users can interfere with one another. This scenario is of particular interest in a CDMA cellular system, in which a given geographical region is divided into many small cells, where the number of cells depends on the size and geometry of the region. Each user has a unique pseudonoise (PN) sequence and communicates through central base-stations with other users in the same cell or different cells. The basestation is assumed to control the average transmission power within its particular cell, to overcome the near/far problem.

The principal purpose of this paper is to evaluate the BER performance of a BPSK direct sequence CDMA system which overlays a narrow-band BPSK waveform and which thus employs a suppression filter to reject the narrow-band interference. We assume flat Rayleigh fading channels and, although synchronous carrier recovery is a difficult task in a fading environment, we assume coherent demodulation.

This paper is organized as follows: The system and channel models are given in Section II. Section III deals with the BER performance of CDMA system in the presence of the overlay. This is followed by some numerical results in Section IV, and finally conclusions are presented in Section V.

\section{SYSTEM MODEL}

Let $K$ denote the number of active users. The transmitted signal of the $k$ th mobile user $(1 \leq k \leq K)$ takes the form

$$
S_{k}(t)=\sqrt{2 P} b_{k}(t) a_{k}(t) \cos \left(2 \pi f_{o} t+\theta_{k}\right)
$$

where $P$ and $f_{o}$ denote the transmitted power and the carrier frequency, respectively, and are common to all users; $\theta_{k}$ is the phase angle introduced by the $k$ th PSK modulator. The $k$ th data signal $b_{k}(t)$ is the $k$ th source information sequence with a rate $1 / T_{b}$. The $\lambda$ th square pulse of $b_{k}(t)$ has amplitude $b_{k}^{(\lambda)}$, taking values form $\{+1,-1\}$ with equal probability. The spreading sequence waveform $a_{k}(t)$ has a rate $1 / T_{c}$ and is modeled as a random binary sequence. The $n$th chip of $a_{k}(t)$ has amplitude $a_{k}^{(n)}$ and takes values from $\{+1,-1\}$. The autocorrelation function $\rho_{T_{c}}(\tau)$ of $a_{k}(t)$ is given by

$$
\rho_{T_{c}}(\tau)= \begin{cases}1-\frac{|\tau|}{T_{c}}, & |\tau| \leq T_{c} \\ 0, & |\tau| \geq T_{c}\end{cases}
$$

We assume that there are $N$ chips (processing gain) of the random sequence in each data bit $\left(T_{b}=N T_{c}\right)$. Therefore, the spread-spectrum system bandwidth $\left(B_{s}\right)$ equals $2 T_{c}^{-1}$. 


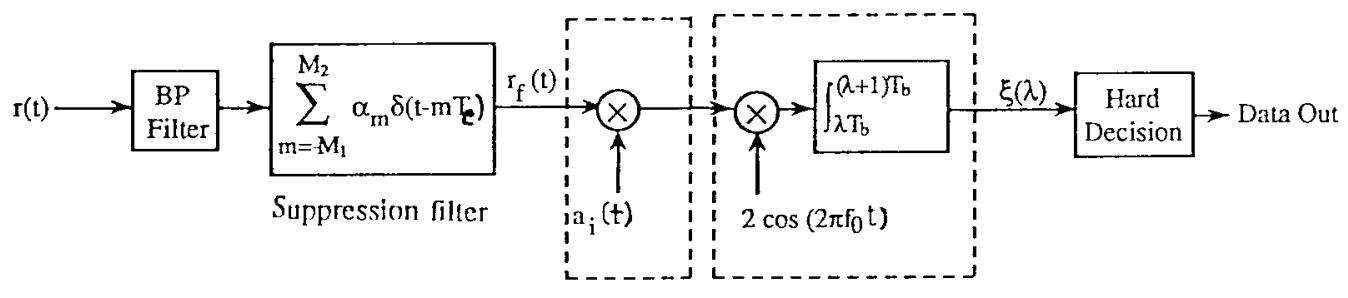

DS despreader PSK demodulator

Fig. 1. Receiver model of a DS system.

We assume that the channel between the $k$ th transmitter and the corresponding receiver at a central station is a frequencynonselective Rayleigh fading channel, and is characterized by three random variables: $\beta_{k}, \tau_{k}$, and $\mu_{k}$, which are, respectively, defined as the gain, delay, and phase of the $k$ th signal at the receiver. The gain $\beta_{k}$ is an independent Rayleigh random variable with parameter $\rho=\rho_{k}=E\left[\beta_{k}^{2}\right] / 2$ for all $k$, while the delay $\tau_{k}$, also independent for each signal, has a uniform distribution in $\left[0, T_{b}\right]$. Furthermore, we assume that the phase $\mu_{k}$ is an independent random variable, uniformly distributed in $[0,2 \pi]$.

The noise $n(t)$ at the input of the receiver is assumed to be additive white Gaussian noise (AWGN) with two-sided power spectral density of $N_{o} / 2$. The interference $J(t)$ is assumed to be narrow-band BPSK signal and is given by

$$
J(t)=\sqrt{2 J} j(t) \cos \left[2 \pi\left(f_{o}+\Delta\right) t+\eta\right]
$$

where $\Delta$ stands for the offset of the interference carrier frequency from the carrier frequency of CDMA signals. $J$ and $\eta$ denote the received interference power and phase, respectively. The information sequence $j(t)$ has a bit rate $1 / T_{j}$, where $T_{j}$ denotes the duration of one bit. The autocorrelation function $\rho_{T_{j}}(\tau)$ of $j(t)$ is given by (2) with $T_{c}$ replaced by $T_{j}$. Therefore, the interference bandwidth is $B_{j}=2 T_{j}^{-1}$ (we assume $B_{j}<B_{s}$ ). An important quantity is the ratio of the interference bandwidth to the spread-spectrum system bandwidth

$$
p=\frac{B_{j}}{B_{s}}=\frac{T_{c}}{T_{j}} .
$$

Therefore, the received signal $r(t)$ at a base station can be represented as

$$
\begin{aligned}
r(t)= & \sqrt{2 P} \sum_{k=1}^{K} \beta_{k} b_{k}\left(t-\tau_{k}\right) a_{k}\left(t-\tau_{k}\right) \cos \left(2 \pi f_{o} t+\phi_{k}\right) \\
& +\sqrt{2 J} j(t) \cos \left[2 \pi\left(f_{o}+\Delta\right) t+\eta\right]+n(t)
\end{aligned}
$$

where $\phi_{k}=\theta_{k}-\mu_{k}-2 \pi f_{o} \tau_{k}$. Note that the interference is assumed not to be fading, based on the fact that the transmission of the narrow-band signal is approximately a direct path from a high microwave tower (or the top of a building) to the receiver of a high base station.

As shown in Fig. 1, the receiver model of the directsequence (DS) system consists of a bandpass filter, a transversal filter (suppression filter), a DS despreader, a PSK demodulator, and a hard decision device. The received signal $r(t)$ enters a front-end ideal bandpass (BP) filter which removes out-of-band noise. The BP filter bandwidth (BW) equals the spread-spectrum system $\mathrm{BW} B_{s}\left(B_{s}=2 T_{c}^{-1}\right)$. We assume that the CDMA signals can pass through the filter without distortion in order to simplify the performance analysis. Also, the received narrow-band interference can pass through the BP filter without distortion, because the bandwidth $B_{j}$ is assumed to be less than the BP filter bandwidth $B_{s}$.

The BP filter output signal enters a suppression filter whose impulse response is $\sum_{m=-M_{1}}^{M_{2}} \alpha_{m} \delta\left(t-m T_{c}\right)$, where $\alpha_{o}=1$, $M_{1} \geq 0, M_{2} \geq 0$, and $M_{1}$ and $M_{2}$ stand for the numbers of taps on the left and right sides, respectively, of the center tap. For $M_{1} \neq 0$ and $M_{2} \neq 0$, the filter is a double-sided suppression filter, whereas for $M_{1}=0$ and $M_{2} \neq 0$ or $M_{1} \neq 0$ and $M_{2}=0$, the filter is a single-sided suppression filter. The suppression filter uses the past and/or future values of the BP filter output signal to predict its current value, and then subtracts the predicted value from its current (actual) value. The suppression filter output is given by

$$
\begin{aligned}
r_{f}(t)= & \sum_{m=-M_{1}}^{M_{2}} \alpha_{m} \\
& \cdot\left\{\sqrt{2 P} \sum_{k=1}^{K} \beta_{k} b_{k}\left(t-\tau_{k}-m T_{c}\right)\right. \\
& \cdot a_{k}\left(t-\tau_{k}-m T_{c}\right) \cos \left(2 \pi f_{o} t+\phi_{k, m}\right) \\
& +\sqrt{2 J} j\left(t-m T_{c}\right) \cos \left[2 \pi\left(f_{o}+\Delta\right)\left(t-m T_{c}\right)+\eta\right] \\
& \left.+\hat{n}\left(t-m T_{c}\right)\right\}
\end{aligned}
$$

where $\hat{n}(t)$ is the filtered AWGN.

The suppression filter signal $r_{f}(t)$ enters a DS despreader. We assume that user $i$ is the reference user. The DS despreader output signal enters a conventional coherent PSK demodulator corresponding to user $i$. The output of the demodulator is a low-pass signal and gives rise to the decision variable $\xi(\lambda)$, where $\lambda$ denotes the $\lambda$ th data bit of the reference user.

\section{SYSTEM PERFORMANCE}

The decision variable $\xi(\lambda)$ can be written as

$$
\xi(\lambda)=\int_{\lambda T_{b}}^{(\lambda+1) T_{b}} r_{f}(t) \cdot a_{i}(t) \cdot 2 \cos \left(2 \pi f_{o} t\right) d t
$$

where $2 \cos \left(2 \pi f_{o} t\right)$ is the recovered carrier and $a_{i}(t)$ is the spreading sequence of the reference user. It is assumed that 
$\phi_{i}=0$ and $\tau_{i}=0$ without loss of generality. Since the highfrequency terms are removed by an integrator following the mixer of the PSK demodulator, the above expression reduces to

$$
\xi(\lambda)=D(\lambda)+F(\lambda)+N(\lambda)+J(\lambda)+\sum_{\substack{k=1 \\ k \neq i}}^{K} I_{k, i}
$$

where the terms on the right-hand side of (8) are described in detail below.

$D(\lambda)$ is the desired signal term of the reference user at the zeroth tap of the suppression filter and is given by

$$
D(\lambda)=\sqrt{2 P} \beta_{i} T_{b} b_{i}^{(\lambda)} .
$$

Given $\beta_{i}$, the useful signal power is equal to

$$
\left.S\right|_{\beta_{i}}=2 P \beta_{i}^{2} T_{b}^{2} \text {. }
$$

$F(\lambda)$ is an internal interference term, due to the reference user, which is caused by the taps of the suppression filter excluding the zeroth tap, and is given by

$$
\begin{aligned}
F(\lambda)= & \sqrt{2 P} \beta_{i} \sum_{\substack{m=-M_{1} \\
m \neq 0}}^{M_{2}} \alpha_{m} \cos \left(\phi_{m}\right) \int_{\lambda T_{b}}^{(\lambda+1) T_{b}} \\
& \cdot b_{i}\left(t-m T_{c}\right) a_{i}\left(t-m T_{c}\right) a_{i}(t) d t \\
= & \sqrt{2 P} \beta_{i} \sum_{\substack{m=-M_{1} \\
m \neq 0}}^{M_{2}} \alpha_{m} \cos \left(\phi_{m}\right) \int_{0}^{T_{b}} \\
& \cdot b_{i}\left(t+\lambda T_{b}-m T_{c}\right) a_{i}\left(t-m T_{c}\right) a_{i}(t) d t \\
= & \sqrt{2 P} \beta_{i} T_{c} \\
& \cdot\left\{\sum_{m_{1}=-M_{1}}^{-1} \alpha_{m} \cos \left(\phi_{m_{1}}\right)\right. \\
& \cdot\left[b_{i}^{(\lambda)} C_{i, i}\left(m_{1}\right)+b_{i}^{(\lambda+1)} C_{i, i}\left(N+m_{1}\right)\right] \\
& +\sum_{m_{2}=1}^{M_{2}} \alpha_{m_{2}} \cos \left(\phi_{m_{2}}\right) \\
& \left.\cdot\left[b_{i}^{(\lambda-1)} C_{i, i}\left(m_{2}-N\right)+b_{i}^{(\lambda)} C_{i, i}\left(m_{2}\right)\right]\right\}
\end{aligned}
$$

where it is assumed that $N \gg M_{1}$ and $N \gg M_{2} . C_{k, i}(l)$ is the discrete-time aperiodic cross-correlation function (or aperiodic autocorrelation function for $k=i) . C_{k, i}(l)$ is defined by [1]

$$
C_{k, i}(l)= \begin{cases}\sum_{j=0}^{N-1-l} a_{k}^{(j)} a_{i}^{(j+l)}, & 0 \leq l \leq N-1 \\ \sum_{j=0}^{N-1+l} a_{k}^{(j-l)} a_{i}^{(j)}, & 1-N \leq l<0 .\end{cases}
$$

It is shown in [3] that

$$
E\left[C_{k, i}(l) C_{k, i}(m)\right]= \begin{cases}N-|l|, & l=|m| \\ 0, & l \neq|m| .\end{cases}
$$

Therefore, the conditional variance of $F(\lambda)$ is given by

$$
\begin{aligned}
\left.\sigma_{1}^{2}\right|_{\beta_{i}}=E\left[\left.F^{2}(\lambda)\right|_{\beta_{i}}\right] & \left\{\sum _ { m _ { 1 } = - M _ { 1 } } ^ { - 1 } \alpha _ { m _ { 1 } } \left[b_{i}^{(\lambda)} C_{i, i}\left(m_{1}\right)\right.\right. \\
= & \left.b_{i}^{(\lambda+1)} C_{i, i}\left(N+m_{1}\right)\right]+\sum_{m_{2}=1}^{M_{2}} \alpha_{m_{2}} \\
& \left.\cdot\left[b_{i}^{(\lambda-1)} C_{i, i}\left(m_{2}-N\right)+b_{i}^{(\lambda)} C_{i, i}\left(m_{2}\right)\right]\right\}^{2} .
\end{aligned}
$$

Using (13), $\left.\sigma_{1}^{2}\right|_{\beta_{i}}$ reduces to

$$
\begin{aligned}
&\left.\sigma_{1}^{2}\right|_{\beta_{i}}=P \beta_{i}^{2} T_{c}^{2}\{ \sum_{m_{1}=-M_{1}}^{-1} \sum_{\hat{m}_{1}=-M_{1}}^{-1} \alpha_{m_{1}} \alpha_{\hat{m}_{1}} E\left[C_{i, i}\left(m_{1}\right)\right. \\
&\left.\cdot C_{i, i}\left(\hat{m}_{1}\right)+C_{i, i}\left(N+m_{1}\right) C_{i, i}\left(N+\hat{m}_{1}\right)\right] \\
&+\sum_{m_{2}=1}^{M_{2}} \sum_{\hat{m}_{2}=1}^{M_{2}} \alpha_{m_{2}} \alpha_{\hat{m}_{2}} \\
& \cdot E\left[C_{i, i}\left(m_{2}-N\right) C_{i, i}\left(\hat{m}_{2}-N\right)\right. \\
&\left.\left.+C_{i, i}\left(m_{2}\right) C_{i, i}\left(\hat{m}_{2}\right)\right]\right\} \\
&=P \beta_{i}^{2} T_{c}^{2}\left[\sum_{m_{1}=-M_{1}}^{-1}\left(\alpha_{m_{1}}^{2} N\right)+\sum_{m_{2}=1}^{M_{2}}\left(\alpha_{m_{2}}^{2} N\right)\right] \\
&=\left(P \beta_{i}^{2} T_{b}^{2} / N\right) \sum_{\substack{M_{2} \\
m=-M_{1} \\
m \neq 0}} \alpha_{m}^{2} .
\end{aligned}
$$

$N(\lambda)$ is due to the noise $\hat{n}(t)$ of the front-end BP filter output. Its variance is given by

$$
\sigma_{2}^{2}=N_{0} T_{b} \sum_{m=-M_{1}}^{M_{2}} \alpha_{m}^{2} .
$$

$J(\lambda)$ is due to the narrow-band interference and is given by

$$
\begin{aligned}
J(\lambda)= & \sum_{m=-M_{1}}^{M_{2}} \alpha_{m} \int_{\lambda T_{b}}^{(\lambda+1) T_{b}} \sqrt{2 J} j\left(t-m T_{c}\right) \\
& \cdot \cos \left[2 \pi \Delta\left(t-m T_{c}\right)+\eta\right] a_{i}(t) d t \\
= & \sum_{m=-M_{1}}^{M_{2}} \alpha_{m} \int_{0}^{T_{b}}\left\{\sqrt{2 J} j\left(t+\lambda T_{b}-m T_{c}\right)\right. \\
& \left.\cdot \cos \left[2 \pi \Delta\left(t+\lambda T_{b}-m T_{c}\right)+\eta\right]\right\} a_{i}(t) d t .
\end{aligned}
$$

The variance of $J(\lambda)$ equals

$$
\begin{aligned}
\sigma_{3}^{2}= & E\left[J^{2}(\lambda)\right] \\
= & \sum_{m_{1}=-M_{1}}^{M_{2}} \sum_{m_{2}=-M_{1}}^{M_{2}} \alpha_{m_{1}} \alpha_{m_{2}} \int_{0}^{T_{b}} \int_{0}^{T_{b}} \\
& \cdot E\left[\sqrt{2 J} j\left(t_{1}+\lambda T_{b}-m_{1} T_{c}\right)\right. \\
& \cdot \cos \left[2 \pi \Delta\left(t_{1}+\lambda T_{b}-m_{1} T_{c}\right)+\eta\right]
\end{aligned}
$$




$$
\begin{aligned}
& \cdot \sqrt{2 J} \beta_{j} j\left(t_{2}+\lambda T_{b}-m_{2} T_{c}\right) \\
& \left.\cdot \cos \left[2 \pi \Delta\left(t_{2}+\lambda T_{b}-m_{2} T_{c}\right)+\eta\right] a_{i}\left(t_{1}\right) a_{i}\left(t_{2}\right)\right] d t_{1} d t_{2} \\
= & \sum_{m_{1}=-M_{1}}^{M_{2}} \sum_{m_{2}=-M_{1}}^{M_{2}} \alpha_{m_{1}} \alpha_{m_{2}} \int_{0}^{T_{b}} \int_{0}^{T_{b}} \\
& \cdot J \rho_{T_{i}}\left(t_{1}-t_{2}-m_{1} T_{c}+m_{2} T_{c}\right) \\
& \cdot \cos \left[2 \pi \Delta\left(t_{1}-t_{2}-m_{1} T_{c}+m_{2} T_{c}\right)\right] \rho_{T_{c}}\left(t_{1}-t_{2}\right) d t_{1} d t_{2} \\
= & J \sum_{m_{1}=-M_{1}}^{M_{2}} \sum_{m_{2}=-M_{1}}^{M_{2}} \alpha_{m_{1}} \alpha_{m_{2}} T_{b} \int_{-T_{b}}^{T_{b}} \\
& \cdot \rho_{T_{i}}\left(\tau-m_{1} T_{c}+m_{2} T_{c}\right) \\
& \cdot \cos \left[2 \pi \Delta\left(\tau-m_{1} T_{c}+m_{2} T_{c}\right)\right] \rho_{T_{c}}(\tau)\left(1-\frac{|\tau|}{T_{b}}\right) d \tau \\
= & T_{b}^{2}(J / N) \sum_{m_{1}=-M_{1}}^{M_{2}} \sum_{m_{2}=-M_{1}}^{M_{2}} \alpha_{m_{1}} \alpha_{m_{2}} \sigma_{j}^{2}\left(m_{1}, m_{2}\right)
\end{aligned}
$$

where $\sigma_{j}^{2}\left(m_{1}, m_{2}\right)$ is given by

$$
\begin{aligned}
\sigma_{j}^{2}\left(m_{1}, m_{2}\right)= & \int_{-1}^{1} \operatorname{sign}\left[1-p\left|x N-m_{1}+m_{2}\right|\right] \\
& \cdot \cos \left[2 \pi q\left(x N-m_{1}+m_{2}\right)\right] \\
& \cdot(1-|x| / N)(1-|x|) d x \\
\approx & \int_{-1}^{1} \operatorname{sign}\left[1-p\left|x N-m_{1}+m_{2}\right|\right] \\
& \cdot \cos \left[2 \pi q\left(x N-m_{1}+m_{2}\right)\right] \\
& \cdot(1-|x|) d x, \quad N \gg 1
\end{aligned}
$$

and where $N=T_{b} / T_{c} p=T_{c} / T_{j}$, and $q=\Delta T_{c}$ denotes the ratio of the offset of the interference carrier frequency to the half spread-spectrum bandwidth. $\operatorname{sign}[x]$ is defined as $\operatorname{sign}[x]=x$ or zero for $x \geq 0$ or $x<0$, respectively.

$I_{k, i}$ is the multiple-access interference term due to the $k$ th user $(k \neq i)$ and is given by

$$
\begin{aligned}
I_{k, i}= & \sqrt{2 P} \sum_{m=-M_{1}}^{M_{2}} \alpha_{m} \beta_{k} \cos \left(\phi_{k}\right) \int_{\lambda T_{b}}^{(\lambda+1) T_{b}} \\
& \cdot b_{k}\left(t-\tau_{k}-m T_{c}\right) a_{k}\left(t-\tau_{k}-m T_{c}\right) a_{i}(t) d t \\
= & \sqrt{2 P} \sum_{m=-M_{1}}^{M_{2}} \alpha_{m} \beta_{k} \cos \left(\phi_{k}\right) \int_{0}^{T_{b}} \\
& \cdot b_{k}\left(t+\lambda T_{b}-\tau_{k}-m T_{c}\right) a_{k}\left(t-\tau_{k}-m T_{c}\right) a_{i}(t) d t \\
= & \sqrt{2 P} \beta_{k} \sum_{m=-M_{1}}^{M_{2}} \alpha_{m} \\
& \cdot\left\{b_{k}^{(\hat{\lambda}-1)} R_{k, i}[\tau(m)]+b_{k}^{(\hat{\lambda})} \hat{R}_{k, i}[\tau(m)]\right\} \cos \left(\phi_{k}\right)
\end{aligned}
$$

where $b_{k}^{(\hat{\lambda}-1)}$ and $b_{k}^{(\hat{\lambda})}$ denote two consecutive bits of the $k$ th user, and $\tau(m)$ is $\tau_{k}+m T_{c}, \bmod T_{b}=N T_{c}$. It is obvious that $\tau(m)$ is uniformly distributed in $\left[0, T_{b}\right] . R_{k, i}(\tau)$ and $\hat{R}_{k, i}(\tau)$ are known as the continuous-time partial cross-correlation functions [1], [2], defined as, respectively,

$$
\begin{aligned}
\hat{R}_{k, i}(\tau)= & \int_{0}^{\tau} a_{k}(t-\tau) a_{i}(t) d t \\
= & C_{k, i}(l-N)\left[(l+1) T_{c}-\tau\right] \\
& +C_{k, i}(l+1-N)\left(\tau-l T_{c}\right)
\end{aligned}
$$

and

$$
\begin{aligned}
\hat{R}_{k, i}(\tau)= & \int_{\tau}^{T_{b}} a_{k}(t-\tau) a_{i}(t) d t \\
= & C_{k, i}(l)\left[(l+1) T_{c}-\tau\right] \\
& +C_{k, i}(l+1)\left(\tau-l T_{c}\right)
\end{aligned}
$$

where $C_{k, i}(l)$ is defined by (12) and $l$ stands for the integer portion of $\tau / T_{c}$. That is, $l=\operatorname{int}\left(\tau / T_{c}\right)$, or $l T_{c} \leq \tau \leq(l+1) T_{c}$.

The variance of $I_{k, i}$ is given by

$$
\begin{aligned}
\sigma_{4}^{2}= & 2 P \rho \sum_{m_{1}=-M_{1}}^{M_{2}} \sum_{m_{2}=-M_{1}}^{M_{2}} \alpha_{m_{1}} \alpha_{m_{2}} \\
& \cdot\left\{E\left[R_{k, i}\left[\tau\left(m_{1}\right)\right] R_{k, i}\left[\tau\left(m_{2}\right)\right]\right]\right. \\
& \left.+E\left[\hat{R}_{k, i}\left[\tau\left(m_{1}\right)\right] \hat{R}_{k, i}\left[\tau\left(m_{2}\right)\right]\right]\right\} .
\end{aligned}
$$

Consider first $E\left[R_{k, i}\left[\tau\left(m_{1}\right)\right] R_{k, i}\left[\tau\left(m_{2}\right)\right]\right]$

$$
\begin{aligned}
E\left[R_{k, i}[\right. & \left.\left.\tau\left(m_{1}\right)\right] R_{k, i}\left[\tau\left(m_{2}\right)\right]\right] \\
=E\{[ & C_{k, i}\left(l_{1}-N\right)\left[\left(l_{1}+1\right) T_{c}-\tau\left(m_{1}\right)\right] \\
& \left.+C_{k, i}\left(l_{1}+1-N\right)\left[\tau\left(m_{1}\right)-l_{1} T_{c}\right]\right] \\
& \cdot\left[C_{k, i}\left(l_{2}-N\right)\left[\left(l_{2}+1\right) T_{c}-\tau\left(m_{2}\right)\right]\right. \\
& \left.\left.+C_{k, i}\left(l_{2}+1-N\right)\left[\tau\left(m_{2}\right)-l_{2} T_{c}\right]\right]\right\}
\end{aligned}
$$

where $l_{1}$ and $l_{2}$ equal $\operatorname{int}\left[\tau\left(m_{1}\right) / T_{c}\right]$ and $\operatorname{int}\left[\tau\left(m_{2}\right) / T_{c}\right]$, respectively, and are uniformly distributed in $[0, N-1]$ because $\tau\left(m_{1}\right)$ and $\tau\left(m_{2}\right)$ have uniform distributions in $\left[0, T_{b}\right]$ $=\left[0, N T_{c}\right]$. For $\tau\left(m_{1}\right)=\tau_{k}+m_{1} T_{c}$ and $\tau\left(m_{2}\right)=\tau_{k}+m_{2} T_{c}$, both $\bmod T_{b}=N T_{c}$, we have $\tau\left(m_{1}\right)=\tau\left(m_{2}\right)+\left(m_{2}-m_{1}\right) T_{c}$, $\bmod T_{b}$. That is, $l_{1}=l_{2}+m_{2}-m_{1}, \bmod N$. Also, $\left[\left(l_{1}+1\right) T_{c}-\right.$ $\left.\tau\left(m_{1}\right)\right]$ and $\left[\left(l_{2}+1\right) T_{c}-\tau\left(m_{2}\right)\right]$ are uniformly distributed in $\left[0, T_{c}\right]$, and $\left[\left(l_{1}+1\right) T_{c}-\tau\left(m_{1}\right)\right]=\left[\left(l_{2}+1\right) T_{c}-\tau\left(m_{2}\right)\right]=\delta$. Equivalently, $\tau\left(m_{1}\right)-l_{1} T_{c}=\tau\left(m_{2}\right)-l_{2} T_{c}=T_{c}-\delta$, which is independent of both $m_{1}$ and $m_{2}$. Thus, when $m_{1}=m_{2}$ (or $l_{1}=l_{2}$ ), using (13), the above expression reduces to

$$
\begin{aligned}
\left.E\left[R_{k, i}\left[\tau\left(m_{1}\right)\right] R_{k, i}\left[\tau\left(m_{2}\right)\right]\right]\right|_{m_{1}=m_{2}} & =E\left\{\left[C_{k, i}(l-N)\right]^{2}\left[(l+1) T_{c}-\tau(m)\right]^{2}\right. \\
& \left.\quad+\left[C_{k, i}(l+1-N)\right]^{2}\left[\tau(m)-l T_{c}\right]^{2}\right\} \\
= & E\left[l \delta^{2}+(l+1)\left(T_{c}-\delta\right)^{2}\right] \\
= & \frac{1}{N} \sum_{l=0}^{N-1} l\left[\frac{1}{T_{c}} \int_{0}^{T_{c}} \delta^{2} d \delta\right] \\
& +\frac{1}{N} \sum_{l=0}^{N-1}(l+1)\left[\frac{1}{T_{c}} \int_{0}^{T_{c}}\left(T_{c}-\delta\right)^{2} d \delta\right] \\
= & \frac{1}{N}\left[\frac{N}{2}(N-1) T_{c}^{2} / 3\right]+\frac{1}{N}\left[\frac{N}{2}(N+1) T_{c}^{2} / 3\right] \\
= & N T_{c}^{2} / 3 \\
= & T_{b}^{2} /(3 N) .
\end{aligned}
$$


When $m_{2}=m_{1}+1$ (or $\left.l_{2}=l_{1}+1\right)$, (24) reduces to

$$
\begin{aligned}
E & {\left.\left[R_{k, i}\left[\tau\left(m_{1}\right)\right] R_{k, i}\left[\tau\left(m_{2}\right)\right]\right]\right|_{m_{2}=m_{1}+1} } \\
& \left.=E\left[\left[C_{k, i}(l+1-N)\right]^{2} \cdot \delta(1-\delta)\right]\right] \\
& =E[(l+1) \cdot \delta(1-\delta)] \\
& =\frac{N+1}{12} T_{c}^{2} \\
& \approx T_{b}^{2} /(12 N) .
\end{aligned}
$$

When $m_{2}=m_{1}-1$ or $l_{2}=l_{1}-1$, the same results as for $m_{2}=m_{1}+1$ apply, and

$$
\left.E\left[R_{k, i}\left[\tau\left(m_{1}\right)\right] R_{k, i}\left[\tau\left(m_{2}\right)\right]\right]\right|_{m_{2}=m_{1}-1}=T_{b}^{2} /(12 N) .
$$

When $\left|m_{2}-m_{1}\right| \geq 2$

$$
\left.E\left[R_{k, i}\left[\tau\left(m_{1}\right)\right] R_{k, i}\left[\tau\left(m_{2}\right)\right]\right]\right|_{\left|m_{2}-m_{1}\right| \geq 2}=0 .
$$

Also, identical results can be obtained for $E\left[\hat{R}_{k, i}\left[\tau\left(m_{1}\right)\right] \hat{R}_{k, i}\left[\tau\left(m_{2}\right)\right]\right] . \quad$ Therefore, $\quad \sigma_{4}^{2} \quad$ is given by

$$
\sigma_{4}^{2}=2 P \rho T_{b}^{2}\left\{\frac{2}{3 N} \sum_{m=-M_{1}}^{M_{2}} \alpha_{m}^{2}+\frac{1}{3 N} \sum_{m=-M_{1}}^{M_{2}} \alpha_{m} \alpha_{m+1}\right\}
$$

where $\alpha_{M_{2}+1}$ is defined to be $\alpha_{-M_{1}}$.

\section{A. Bit Error Rate}

Collecting the above results, the decision variable $\xi(\lambda)$, given by (8), consists of a useful term $D(\lambda)$ and a total interference term [internal interference $F(\lambda)+$ Gaussian noise $N(\lambda)+$ narrow-band interference $J(\lambda)+$ multiple-access interference $\left.\sum_{k=1, k \neq i}^{K} I_{k, i}\right]$. The total interference has the conditional variance, given $\beta_{i}$, of

$$
\begin{aligned}
\left.\sigma^{2}\right|_{\beta_{i}}= & \left.\sigma_{1}^{2}\right|_{\beta_{i}}+\sigma_{2}^{2}+\sigma_{3}^{2}+\sigma_{4}^{2}(K-1) \\
= & P \beta_{i}^{2}\left(T_{b}^{2} / N\right) \sum_{\substack{m=-M_{1} \\
m \neq 0}}^{M_{2}} \alpha_{m}^{2}+N_{0} T_{b} \sum_{m=-M_{1}}^{M_{2}} \alpha_{m}^{2} \\
& +T_{b}^{2}(J / N) \cdot \sum_{m_{1}=-M_{1}}^{M_{2}} \sum_{m_{2}=-M_{1}}^{M_{2}} \alpha_{m_{1}} \alpha_{m_{2}} \sigma_{j}^{2}\left(m_{1}, m_{2}\right) \\
& +(K-1) 2 P \rho T_{b}^{2}\left[\frac{2}{3 N} \sum_{m=-M_{1}}^{M_{2}} \alpha_{m}^{2}\right. \\
& \left.\quad+\frac{1}{3 N} \sum_{m=-M_{1}}^{M_{2}} \alpha_{m} \alpha_{m+1}\right]
\end{aligned}
$$

where $\sigma_{j}^{2}\left(m_{1}, m_{2}\right)$ is given by (19).

When the number $(K)$ of active users is large, it is shown in the Appendix that, according to the central-limit theorem, given $\beta_{i}$, the sum of the internal interference term $F(\lambda)$, the narrow-band interference term $J(\lambda)$, and the multiple- access interference terms $\sum_{k \neq i} I_{k, i}$ can be approximated by a conditional Gaussian random variable. Hence, given $\beta_{i}$, all interference terms and the noise term can be combined into a single conditional Gaussian random variable. Therefore, the conditional signal-to-noise ratio of the decision variable $\xi(\lambda)$ is given by

$$
r_{b}\left(\beta_{i}^{2}\right)=\frac{\left.S\right|_{\beta_{i}}}{\left.2 \sigma^{2}\right|_{\beta_{i}}} .
$$

The conditional BER is given by

$$
P_{e}\left(\beta_{i}^{2}\right)=\frac{1}{\sqrt{2 \pi}} \int_{\sqrt{2 r_{b}\left(\beta_{i}^{2}\right)}}^{\infty} e^{-x^{2} / 2} d x
$$

To obtain the average BER, we must average $P_{e}\left(\beta_{i}^{2}\right)$ over the probability density function of $\beta_{i}^{2}$. That is, we must evaluate the integral

$$
P_{e}=\int_{0}^{\infty} P_{e}\left(\beta_{i}^{2}\right) p\left(\beta_{i}^{2}\right) d\left(\beta_{i}^{2}\right)=\int_{0}^{\infty} P_{e}(r) P(r) d r
$$

where $r=\beta_{i}^{2}$ has a chi-square distribution, given by

$$
P\left(\beta_{i}^{2}\right)=p(r)=\frac{1}{\rho} e^{-r / \rho}, \quad r>0
$$

where $\rho=E\left[\beta_{i}^{2}\right] / 2$.

By numerical integration, using the expression in (33), the average BER can be obtained. However, it is cumbersome to evaluate the average BER from (33), because of the 2-D numerical integration involved. An alternative is to evaluate the average BER in closed form using an approximation in (30). It is clearly seen from (30) that when the number of active users $K$ is large $(K \gg 1)$, the variance of the internal interference term $F(\lambda)$ is much less than of the total multiple-access interference term $\sum_{k \neq i} I_{k, i}$. That is, $E\left[\sigma_{1}^{2} \mid \beta_{i}\right] \ll(K-1) \sigma_{4}^{2}$, so that $F(\lambda)$ can be safely ignored in order to obtain a simple BER analytical result. Therefore, the variance of the total interference and noise is independent of $\beta_{i}$, and is approximated by

$$
\begin{aligned}
\sigma^{2}= & N_{0} T_{b} \sum_{m=-M_{1}}^{M_{2}} \alpha_{m}^{2}+T_{b}^{2}(J / N) \\
& \cdot \sum_{m_{1}=-M_{1}}^{M_{2}} \sum_{m_{2}=-M_{1}}^{M_{2}} \alpha_{m_{1}} \alpha_{m_{2}} \sigma_{j}^{2}\left(m_{1}, m_{2}\right) \\
& +(K-1) 2 P \rho T_{b}^{2} \\
& \cdot\left[\frac{2}{2 N} \sum_{m=-M_{1}}^{M_{2}} \alpha_{m}^{2}+\frac{1}{3 N} \sum_{m=-M_{1}}^{M_{2}} \alpha_{m} \alpha_{m+1}\right] .
\end{aligned}
$$

Given $\beta_{i}$, the signal-to-noise ratio now becomes

$$
\hat{r}_{b}\left(\beta_{i}^{2}\right)=\left.S\right|_{\beta_{i}} / 2 \sigma^{2}
$$

Replacing $r_{b}\left(\beta_{i}^{2}\right)$ with $\hat{r}_{b}\left(\beta_{i}^{2}\right)$ in (32), we carry out the integration in (33). The result of the integration, from [15], is given by

$$
\hat{p}_{e}=\frac{1}{2}\left[1-\sqrt{\frac{r_{b}}{1+r_{b}}}\right] \approx \frac{1}{4 r_{b}}
$$


where $r_{b}$ denotes the average signal-to-noise ratio (SNR), with "noise" taken to be the sum of the contributions due to the narrow-band interference, the multiple-access interference, and the thermal noise and is given by

$$
\begin{aligned}
r_{b}= & \frac{1}{2} \frac{E\left[\left.S\right|_{\beta_{i}}\right]}{\sigma^{2}} \\
= & \frac{2 P \rho T_{b}^{2}}{\sigma^{2}} \\
= & \left\{\left[\frac{\bar{E}_{b}}{N_{o}}\right]^{-1} \sum_{m=-M_{1}}^{M_{2}} \alpha_{m}^{2}+\frac{1}{N}\left[\frac{J}{S}\right]\right. \\
& \quad \sum_{m=-M_{1}}^{M_{2}} \sum_{m=-M_{1}}^{M_{2}} \alpha_{m_{1}} \alpha_{m_{2}} \sigma_{j}^{2}\left(m_{1}, m_{2}\right) \\
& \left.\quad \frac{(K-1)}{3 N}\left[\sum_{m=-M_{1}}^{M_{2}} 2 \alpha_{m}^{2}+\sum_{m=-M_{1}}^{M_{2}} \alpha_{m} \alpha_{m+1}\right]\right\}^{-1} .
\end{aligned}
$$

In (38), $\bar{E}_{b}=2 P \rho T_{b}$ is the average energy-per-bit and $J / S=J /(2 P \rho)$ denotes the interference power to signal power ratio. The accuracy of the above approximation will be demonstrated in the next section.

\section{B. Determination of the Suppression Filter Coefficients}

The coefficients of the suppression filter can be determined by solving the following Wiener-Hopf equations [8]:

$$
\begin{aligned}
& \sum_{\substack{m=-M_{1} \\
m \neq 0}}^{M_{2}} \alpha_{m} R\left[(n-m) T_{c}\right]+R\left(n T_{c}\right) \\
& \quad n=0, \quad n=-M_{1}, \cdots,-1,1, \cdots, M_{2}, \quad n \neq 0 .
\end{aligned}
$$

In (39), $R(\tau)$ is the autocorrelation function of the input signal of the suppression filter and can be expressed as

$$
R(\tau)=\frac{1}{2} \rho(\tau) \cos \left(2 \pi f_{\circ} \tau\right)
$$

where $\rho(\tau)$ is the low-pass version of $R(\tau)$. Therefore, for $f_{0} T_{c}$ an integer, the Wiener-Hopf equations become

$$
\begin{aligned}
& \sum_{\substack{m=-M_{1} \\
m \neq 0}}^{M_{2}} \alpha_{m} \rho\left[(n-m) T_{c}\right]+\rho\left(n T_{c}\right) \\
& \quad=0, \quad n=-M_{1}, \cdots,-1,1, \cdots, M_{2}, \quad n \neq 0 .
\end{aligned}
$$

$\rho\left(l T_{c}\right)$ can be written as

$$
\rho\left(l T_{c}\right)=\rho_{s}\left(l T_{c}\right)+\rho_{\hat{n}}\left(l T_{c}\right)+\rho_{j}\left(l T_{c}\right)
$$

where $\rho_{s}\left(l T_{c}\right), \rho_{\hat{n}}\left(l T_{c}\right)$, and $\rho_{j}\left(l T_{c}\right)$ are, respectively, the lowpass versions of the DS signal, noise and interference functions of the input signal to the suppression filter. The DS term is given by

$$
\begin{aligned}
\rho_{s}\left(l T_{c}\right)= & \frac{1}{2} E\left\{\left[\sqrt{2 P} \sum_{k_{1}=1}^{K} \beta_{k_{1}} b_{k_{1}}\left(t-\tau_{k_{1}}\right) \cdot a_{k_{1}}\left(t-\tau_{k_{1}}\right)\right]\right. \\
& \cdot\left[\sqrt{2 P} \sum_{k_{2}=1}^{K} \beta_{k_{2}} b_{k_{2}}\left(t+l T_{c}-\tau_{k_{2}}\right)\right. \\
\cdot & \left.\left.a_{k_{2}}\left(t+l T_{c}-\tau_{k_{2}}\right)\right]\right\} \\
= & P \cdot \sum_{K_{1}=1}^{K} \sum_{K_{2}=1}^{K} E\left[\beta_{k_{1}} \beta_{k_{2}}\right] \\
& \cdot E\left[b_{k_{1}}\left(t-\tau_{k_{1}}\right) b_{k_{2}}\left(t+l T_{c}-\tau_{k_{2}}\right)\right] \\
& \cdot E\left[a_{k 1}\left(t-\tau_{k 1}\right) a_{k 2}\left(t+l T_{c}-\tau_{k 2}\right)\right] \\
= & P \cdot \sum_{k=1}^{K} E\left[\beta_{k}^{2}\right] E\left[b_{k}\left(t-\tau_{k}\right) b_{k}\left(t+l T_{c}-\tau_{k}\right)\right] \\
& \cdot E\left[a_{k}\left(t-\tau_{k}\right) a_{k}\left(t+l T_{c}-\tau_{k}\right)\right] .
\end{aligned}
$$

Since $E\left[a_{k_{1}}\left(t-\tau_{k_{1}}\right) a_{k_{2}}\left(t+l T_{c}-\tau_{k_{2}}\right)\right]=0$ for $k_{1} \neq k_{2}, \rho_{T_{c}}(\tau)$ is given by (2), and further $E\left[a_{k}\left(t-\tau_{k}\right) a_{k}\left(t+l T_{c}-\tau_{k}\right)\right]$ is given by

$$
E\left[a_{k}\left(t-\tau_{k}\right) a_{k}\left(t+l T_{c}-\tau_{k}\right)\right]= \begin{cases}1, & l=0 \\ 0, & l \neq 0\end{cases}
$$

one obtains

$$
\rho_{s}\left(l T_{c}\right)= \begin{cases}2 K P \rho, & l=0 \\ 0, & l \neq 0 .\end{cases}
$$

The filtered-noise term $\hat{n}(t)$ has a flat power spectral density of $N_{0} / 2$ with the bandwidth of $2 / T_{c}$. Since the autocorrelation function of a signal is the Fourier transform of its power spectral density function, the low-pass version of the autocorrelation of the noise term is given by

$$
\begin{aligned}
\rho_{\hat{n}}\left(l T_{c}\right) & =\frac{N_{0}}{\pi} \frac{\sin \left(\pi B_{c} l T_{c}\right)}{l T_{c}} \\
& =\frac{N_{0}}{\pi} \cdot \frac{\sin (2 \pi l)}{l T_{c}} \\
& = \begin{cases}2 N_{0} / T_{c}, & l=0 \\
0, & l \neq 0 .\end{cases}
\end{aligned}
$$

Since the base-band signal of the narrow-band interference is a binary sequence, its autocorrelation is a triangular function. Therefore, the low-pass version of the autocorrelation of the narrow-band interference is given by

$$
\begin{aligned}
\rho_{j}\left(l T_{c}\right)= & E\{\sqrt{2 J} j(t) \cos (2 \pi \Delta t+\eta) \\
& \left.\cdot \sqrt{2 J} j\left(t+l T_{c}\right) \cos \left(2 \pi \Delta\left(t+l T_{c}\right)+\eta\right)\right\} \\
= & J E\left\{j(t) j\left(t+l T_{c}\right)\right\} \cos \left(2 \pi \Delta l T_{c}\right) \\
= & \begin{cases}J(1-|l| p) \cos (2 \pi l q), & |l| \leq \operatorname{int}[1 / p] \\
0, & |l|>\operatorname{int}[1 / p]\end{cases}
\end{aligned}
$$

where int $[x]$ is defined as the integer part of $x$. Therefore, from the above, one obtains

$$
\rho\left(l T_{c}\right)=2 P \rho \hat{\rho}\left(l T_{c}\right)
$$




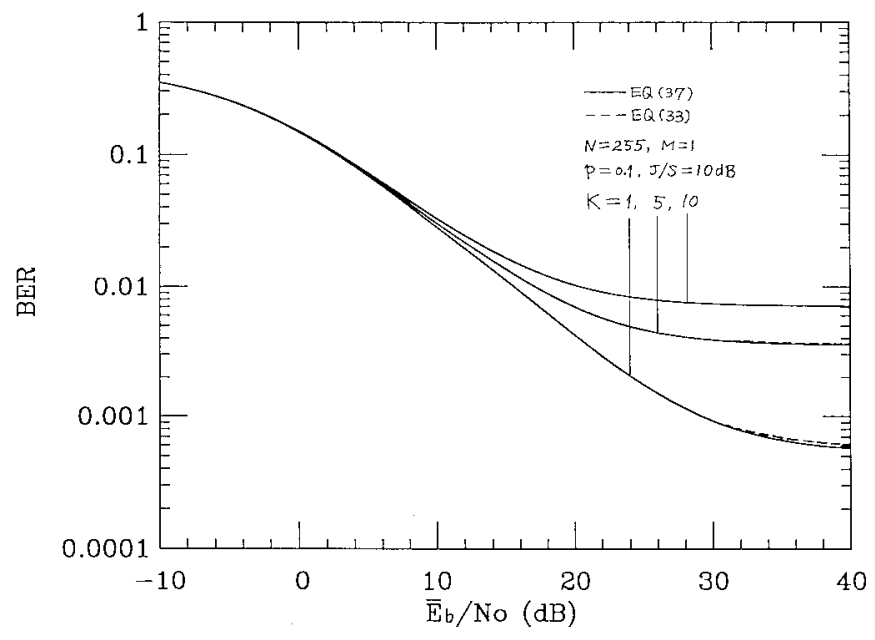

Fig. 2. BER comparison of the system with suppression filters using (33) and (37).

where $\hat{\rho}\left(l T_{c}\right)$ is defined as

$$
\hat{\rho}\left(l T_{c}\right) \equiv \begin{cases}K+2 N\left[\bar{E}_{b} / N_{0}\right]^{-1}+J / S, & l=0 \\ (J / S)(1-|l| p) \cos (2 \pi l q), & |l| \leq \operatorname{int}[1 / p] \\ 0, & |l|>\operatorname{int}[1 / p]\end{cases}
$$

where $S=2 P \rho$ and $\bar{E}=2 P \rho T_{b}$. By replacing $\rho(\tau)$ with $\hat{\rho}(\tau)$ in (41), we can obtain the coefficients $\alpha_{m}$, and then we can obtain the average BER performance.

\section{NUMERICAL RESULTS}

In this section, using the above analytical results, we provide some representative numerical curves illustrating the BER performance of the DS-CDMA system in the presence of narrow-band interference. Unless noted otherwise, it is assumed that the ratio of the offset of the interference carrier frequency to the half spread-spectrum bandwidth $q=0$, that is, the carrier frequencies of the CDMA signals and BPSK narrow-band signal are identical (i.e., $\Delta=0$ ).

In Fig. 2, the BER of the system with a double-sided filter is evaluated by using (33) and (37) for various numbers of users $(K)$. It is seen that, as expected, (33) and (37) are very close for $K \gg 1$. Even for $K=1$, the difference between (33) and (37) is very small, so that (37) can be considered to be an excellent approximation to (33). Therefore, ignoring the internal interference $F(\lambda)$ is reasonable.

Fig. 3 shows the BER performance of the system with a double-sided suppression filter as a function of the signal-tonoise ratio $\bar{E}_{b} / N_{0}$. Also, the BER of the system both with a single-sided suppression filter and without a suppression filter are plotted in order to allow for comparison. The number of active users $K=10$, the number of chips per bit $N=255$, and the interference power-to-signal power ratio $J / S=20$ $\mathrm{dB}$. It is assumed that the two-sided filter has three taps and is symmetric (i.e., $M_{1}=M_{2}=M=1$ ), and the single-sided filter has the same number of taps (i.e., $M_{1}=$ $2 M=2$ and $M_{2}=0$, or $M_{1}=0$ and $M_{2}=2 M=2$ ). Finally, the narrow-band interference occupies $10 \%(p=0.1)$ of the spread-spectrum bandwidth. As expected, the BER

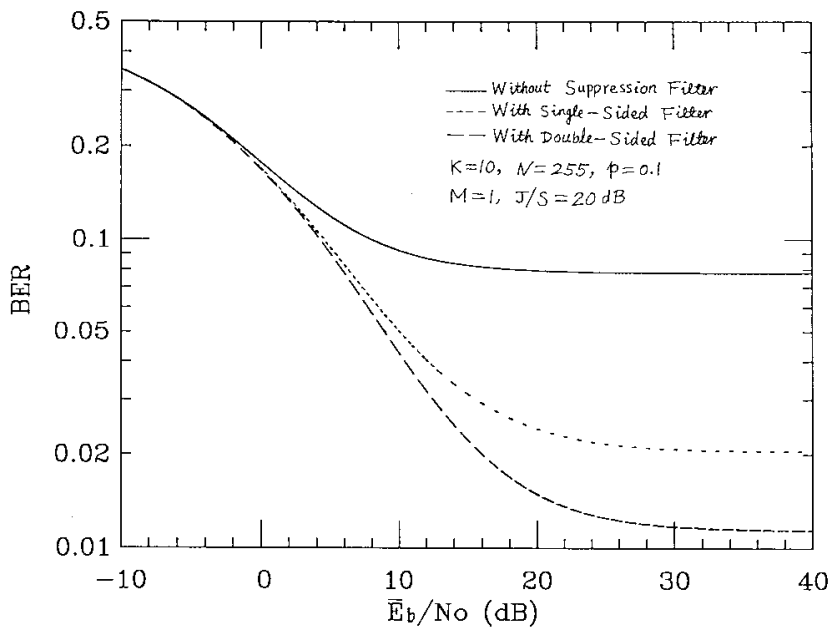

Fig. 3. BER performance of DS-CDMA systems with and without suppression filters.

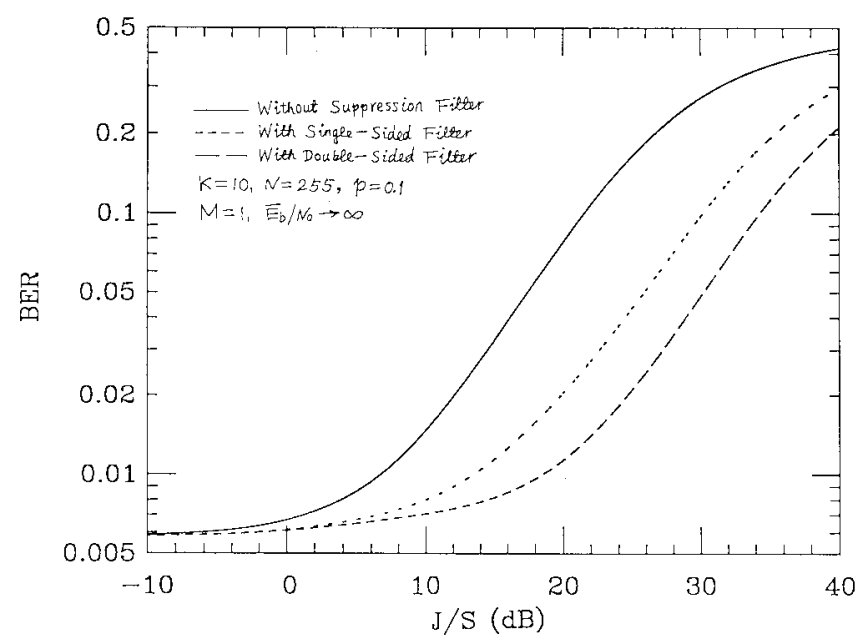

Fig. 4. The asymptotic $\left(\bar{E}_{b} / N_{o} \rightarrow \infty\right)$ BER performance of DS-CDMA systems with and without suppression filters as a function of the interference power-to-signal power ratio $(J / S)$.

performance can be improved by using a suppression filter. In particular, tremendous improvement can be achieved by using a double-sided filter. The double sided filter is superior to the single-sided filter for the same number of the total taps because the former uses the autocorrelation function of the narrow-band interference more efficiently than does the latter.

Fig. 4 illustrates the asymptotic $\left(\bar{E}_{b} / N_{0} \rightarrow \infty\right)$ BER of the system with double-sided filters, single-sided filters, and without suppression filters as a function of $(J / S)$. As expected, the BER's of the systems with or without the suppression filters increase as $J / S$ increases. When $J / S$ is large (i.e., $J / S \geq 10 \mathrm{~dB}$ ) the system without a suppression filter degrades significantly, whereas the system is much more tolerant of the interference when the suppression filter is present.

In Fig. 5, the asymptotic BER of the system with and without the suppression filter is shown as a function of the ratio $(p)$ of the interference bandwidth to the spread-spectrum bandwidth. The system parameters are again $K=10, N=$ 255 , and $J / S=20 \mathrm{~dB}$, and the number of total taps is again 


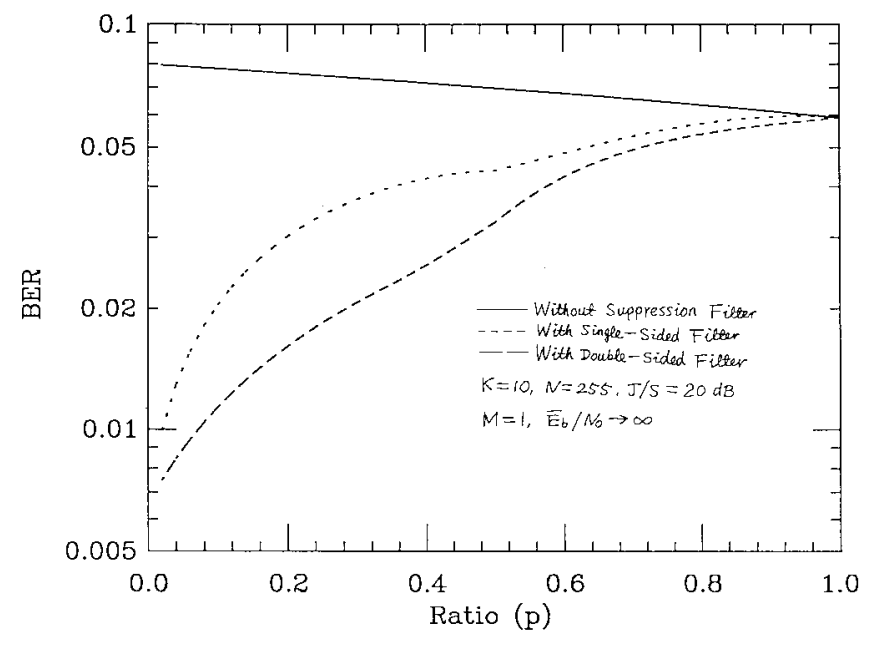

Fig. 5. The asymptotic $\left(\bar{E}_{b} / N_{o} \rightarrow \infty\right)$ BER of DS-CDMA systems with and without suppression filters as a function of the ratio $(p)$ of the interference BW to the spread-spectrum BW.

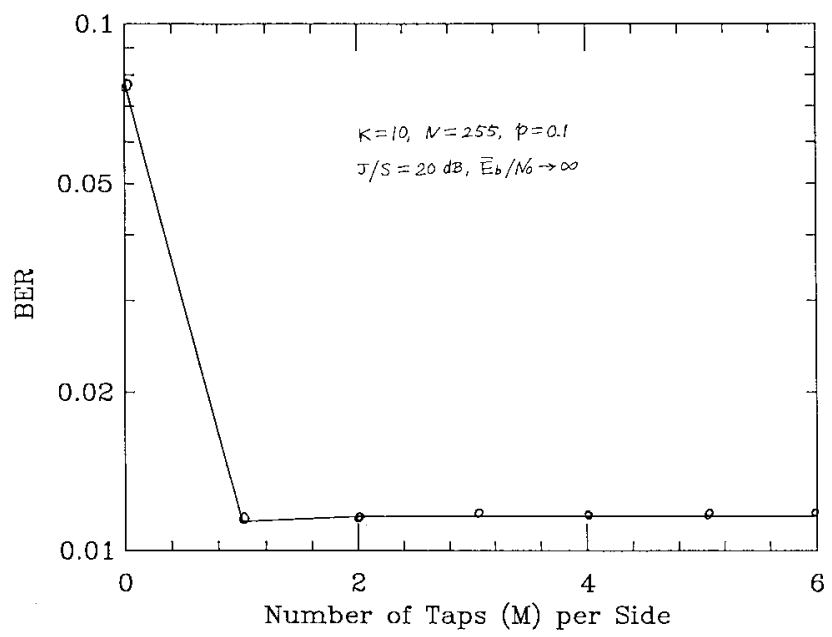

Fig. 6. The asymptotic BER of DS-CDMA systems with double-sided suppression filter as a function of number of taps on each side $\left(M_{1}=M_{2}=M\right)$.

three. It is seen from the figure that the CDMA system with a double-sided filter can suppress the narrow-band interference very well when $p$ is small, but is ineffective in suppressing the interference when $p$ is large. On the other hand, the BER performance of the system without a suppression filter is almost insensitive to the value of $p$, although it becomes slightly better with increasing $p$ for fixed $J / S$. For $p=1$, the BER's are the same for the systems with and without suppression filters. This is because the autocorrelation function of interference, $\rho_{j}\left(l T_{c}\right)$, is zero for $l \neq 0$ (or $\alpha_{m}=0$ for $m \neq 0$ ).

Fig. 6 illustrates the asymptotic BER for a double-sided suppression filter as a function of the number of taps on each side $\left(M_{1}=M_{2}=M\right)$. It is seen from the figure that the BER decreases first when $M=1$ and then keeps a value with increasing $M$. Indeed, for $M_{1}=M_{2}=0$ (without the suppression filter), the narrow-band interference $J / S=20$ $\mathrm{dB}$ is the dominating disturbance; the bit errors are caused by the narrow-band interference. For $M_{1}=M_{2} \geq 1$, the narrow-band interference is reduced by the suppression filter, and increasing $M$ beyond one does not noticeably reduce the narrow-band interference, because $\hat{\rho}\left(T_{c}\right)$ and $\hat{\rho}\left(-T_{c}\right)$ are almost identical to $\hat{\rho}(0)$ in (48), assuming $J / S \gg K$. Thus, the suppression filter with $M_{1}=M_{2}=1$ is sufficient to reject the interference in this case (small $p$ and $q=0$ ), and more taps than one on each side are unnecessary. Table I shows the tap coefficients of the double-sided suppression filters for different numbers of taps each side $\left(M_{1}=M_{2}=0,1,2,3\right)$. As shown in Table I, for $M=0, A \gg B$, where $A$ represents the effect of the narrow-band interference and $B$ represents the effect of the multiple-access interference; in this case, the narrowband interference dominates. However, for $M \geq 1$, neither $A$ nor $B$ varies very much. Therefore, we conclude that when the carrier frequencies of the narrow-band interference and CDMA signals are the same, the suppression filter with three total taps is sufficient to reduce the narrow-band interference, and increasing the number of total taps beyond three is not necessary.

In Fig. 7, the asymptotic BER of the DS-CDMA system with double-sided filters is plotted as a function of active users $(K)$ for different values of $J / S$. The system parameters are $N=255, M_{1}=M_{2}=1$, and $p=0.1$. Also, the BER of the system without the suppression filter is shown for comparison. It is seen that, for a given BER, the system with the doublesided filter can support many more users than can the system without the suppression filter, especially when the $J / S$ is large. When the interference is not too large (i.e., $J / S \leq 10 \mathrm{~dB}$ ) the system employing the suppression filter can support almost the same number of users as can the system in the absence of the interference.

Fig. 8 illustrates the BER performance of the systems with double-sided suppression filters as a function of the ratio $(q)$ of the offset of the interference carrier frequency to the half spread-spectrum bandwidth for different number of the taps on each side. It is seen that when $q$ is very small, the BER's of the system with double-sided filters for $M \geq 1$ are almost identical, as was the case in Fig. 6. However, the BER performance improves as $M$ increases when $q$ increases, and the BER for large $M$ is also more robust to a change in $q$. Furthermore, when $q$ is very large (i.e., $q \geq 90 \%$ ), the suppression filter is unnecessary. Also, for $q \approx 0.25$, the BER's of the systems with the double-sided suppression filter $\left(M_{1}=M_{2}=1\right)$ and without the suppression filter are almost identical, because the low-pass version of the autocorrelation of the interference is zero, (i.e., $\rho_{j}=\left(l T_{c}\right)=0$ for $l \neq 0$ ), so that all off-center tap coefficients are zero.

\section{CONCLUSIONS}

The BER performance of a DS-CDMA system operating over a Rayleigh fading channel, overlaying a narrow-band BPSK waveform, and employing a suppression filter in the receiver, has been evaluated. From the numerical results the following conclusions can be drawn.

1) A large improvement in BER can be achieved by using double-sided suppression filters. The double-sided suppression filter is superior to the single-sided suppression filter for the same number of total taps because 
TABLE I

$\left(K=10, N=255, p=0.1, \bar{E}_{b} / N_{o} \rightarrow \infty, J / S=20 \mathrm{~dB}\right)$

\begin{tabular}{|c|c|c|c|c|c|c|c|c|c|}
\hline \multirow{2}{*}{$\begin{array}{l}\text { Number of taps } \\
\text { each side (M) }\end{array}$} & \multicolumn{7}{|c|}{ Coefficients $\left(\alpha_{\mathrm{m}}\right)$} & \multirow{2}{*}{ A } & \multirow{2}{*}{$\mathrm{B}$} \\
\hline & $\alpha_{-3}$ & $\alpha_{-2}$ & $\alpha_{-1}$ & $\alpha_{0}$ & $\alpha_{1}$ & $\alpha_{2}$ & $\alpha_{3}$ & & \\
\hline 0 & 0 & 0 & 0 & 1 & 0 & 0 & 0 & 0.38 & 0.023 \\
\hline 1 & 0 & 0 & -0.474 & 1 & -0.474 & 0 & 0 & 0.021 & 0.026 \\
\hline 2 & 0 & -0.119 & -0.373 & 1 & -0.373 & -0.119 & 0 & 0.025 & 0.023 \\
\hline 3 & -0.032 & -0.1 & -0.367 & l & -0.367 & -0.1 & -0.032 & 0.026 & 0.023 \\
\hline
\end{tabular}

$$
\begin{aligned}
& A=\frac{1}{N}\left(\frac{J}{S}\right) \sum_{m_{1}=-M}^{M} \sum_{m_{2}=-M}^{M} \alpha_{m_{1}} \alpha_{m_{2}} \sigma_{2}^{2}\left(m_{1}, m_{2}\right) \\
& B=\frac{K-1}{3 N} \sum_{m=-M}^{M} \alpha_{m}\left[2 \alpha_{m}+\alpha_{m+1}\right]
\end{aligned}
$$

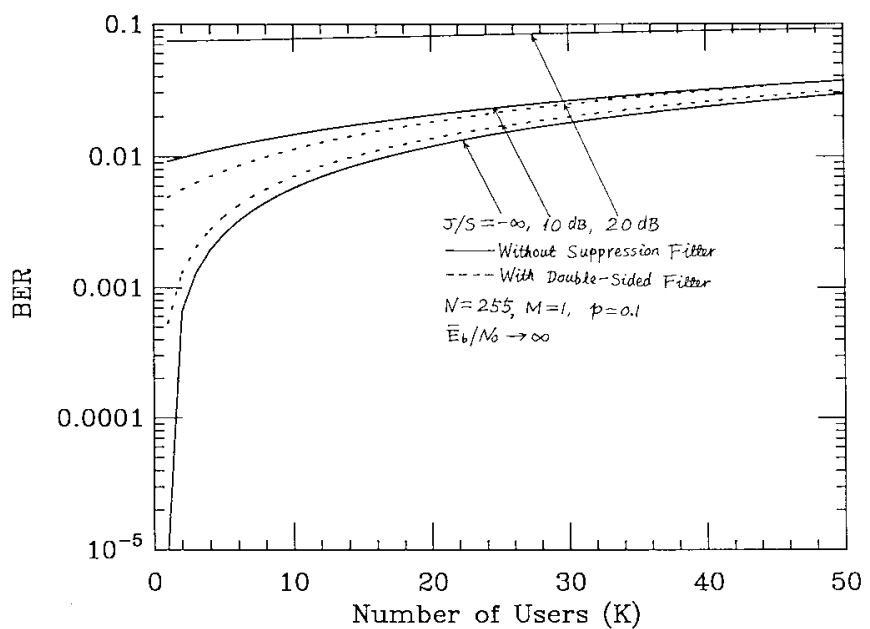

Fig. 7. The asymptotic BER of DS-CDMA systems with and without double-sided filters as a function of the number of active users $(K)$.

the double-sided suppression filter is more effective in using the autocorrelation function of the narrow-band interference in order to predict and subtract out that interference.

2) When the ratio $(q)$ of the interference carrier frequency to half of the spread-spectrum bandwidth is very small, that is, when the interference carrier frequency is very close to the spread-spectrum carrier frequency, the suppression filter with three total taps is sufficient to reduce the narrow-band interference and increasing the number of total taps beyond three is not necessary. However, spread-spectrum systems with a large number of taps (i.e., $M_{1}=M_{2}>1$ ) is preferable when the ratio is large. Further, when the ratio $q$ is greater than $90 \%$, the suppression filter is not useful.

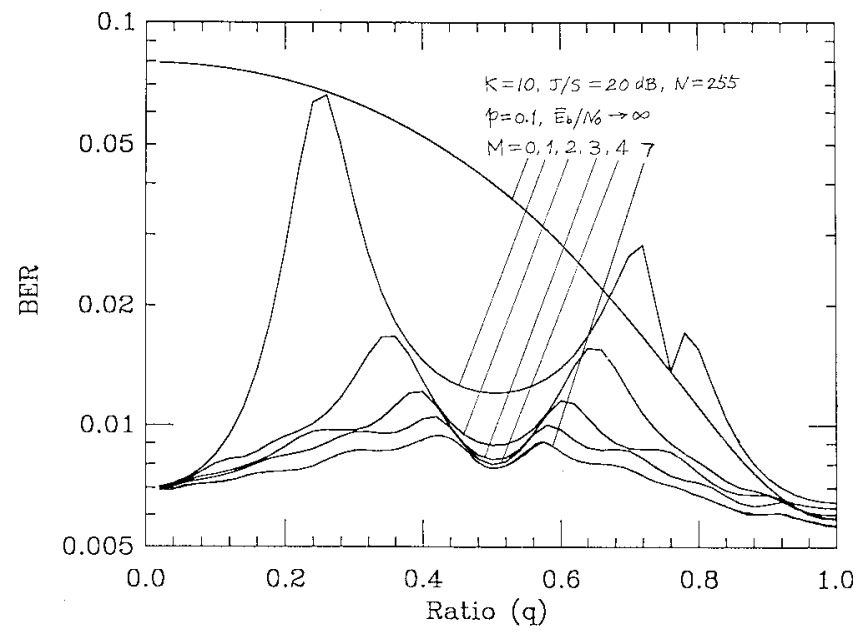

Fig. 8. The asymptotic BER of the system with double-sided filters as a function of the ratio $(q)$ of the interference carrier frequency to the half spread-spectrum BW.

3) When a suppression filter is used, the internal interference, $F(\lambda)$, which is caused by the reference user signal, can be neglected in order to obtain a simple analytical BER result.

\section{APPENDIX}

DERIVATION OF THE GAUSSIAN APPROXIMATION FOR ALL INTERFERENCE TERMS

The random variable due to all interference terms is equal to

$$
G \equiv F(\lambda)+J(\lambda)+\sum_{\substack{k=1 \\ k \neq i}}^{K} I_{k, i} \equiv \sum_{\substack{k=1 \\ k \neq i}}^{K} I_{k, i}
$$


where $F(\lambda), J(\lambda)$, and $I_{k, i}(k=1, \cdots, K, k \neq i)$ are the internal interference, narrow-band interference, and multipleaccess interference terms, respectively, and where $I_{k, i}$ is defined as $F(\lambda)$ for $k=K+1$ and $I_{k, i}$ is defined as $J(\lambda)$ for $k=K+2$. Note that from (11), (17), and (20), all interference terms are functions of the $\left\{a_{i}^{(n)}\right\}$. However, that binary sequence is multiplied by $\left\{a_{i}^{(n-m)}\right\}, m \neq 0$, in $F(\lambda)$, and multiplied by $\left\{a_{k}^{(n)} \tau_{k}+a_{k}^{(n-1)}\left(T_{c}-\tau_{k}\right)\right\}$ in $I_{k, i}$. Since each term of each random binary sequence is an i.i.d. binary random variable, and since all data sequences, time delays and carrier phases are mutually independent, it follows that all $K+1$ interference terms are statistically independent. Further, the statistics (i.e., the moments) of these random variables are finite. Hence, if we define

$$
\left[E\left|I_{k, i}\right|^{3}\right]_{\max } \equiv \max \left\{E\left|I_{k, i}\right|^{3}, k=1, \cdots, k+2, k \neq i\right\}
$$

and

$$
\begin{aligned}
{\left[E\left|I_{k, i}\right|^{2}\right]_{\min } } & \equiv \min \left\{E\left|I_{k, i}\right|^{2}, k=1, \cdots, k+2, k \neq i\right\} \\
& >0
\end{aligned}
$$

then, from the Liapunoff version of the central-limit theorem [16], the condition for asymptotic normality can be expressed in terms of the variable

$$
\begin{aligned}
\gamma & \equiv \frac{\left[\sum_{\substack{k=1 \\
k \neq i}}^{k+2} E\left|I_{k, i}\right|^{3}\right]^{1 / 3}}{\left[\sum_{\substack{k=1 \\
k \neq i}}^{k+2} E\left(I_{k, i}\right)^{2}\right]^{1 / 2}} \leq \frac{\left[\sum_{\substack{k=1 \\
k \neq i}}^{k+2}\left[E\left|I_{k, i}\right|^{3}\right]_{\max }\right]^{1 / 3}}{\left[\sum_{\substack{k=1 \\
k \neq i}}^{k+2}\left[E\left(I_{k, i}\right)^{2}\right]_{\min }\right]^{1 / 2}} \\
= & \frac{(K+1)^{1 / 3}\left[\left[E\left|I_{k, i}\right|^{3}\right]_{\max }\right]^{1 / 3}}{(K+1)^{1 / 2}\left[\left[E\left|I_{k, i}\right|^{2}\right]_{\min }\right]^{1 / 2}} .
\end{aligned}
$$

In particular, since

$$
\frac{\left[\left[E\left|I_{k, i}\right|^{3}\right]_{\max }\right]^{1 / 3}}{\left[\left[E\left(I_{k, i}\right)^{2}\right]_{\min }\right]^{1 / 2}}
$$

is finite, when $K \rightarrow \infty$, we have

$$
\lim _{K \rightarrow \infty} \gamma=0
$$

Therefore, given $\beta_{i}$, the sum of all the interference terms, $G$, can be approximated by a conditional Gaussian random variable.

\section{ACKNOWLEDGMENT}

The author would like to thank Prof. L. B. Milstein, University of California at San Diego, for his helpful suggestions and valuable comments.

\section{REFERENCES}

[1] M. B. Pursley, "Spread spectrum multiple access communications," in Multi-User Communications, G. Longo, Ed. Vienna/New York: Springer-Verlag, 1981, pp. 139-199.

[2] M. B. Pursley, D. V. Sarwate, and W. E. Stark, "Error probability for direct-sequence spread-spectrum multiple-access communications-Part I: Upper and lower bounds," IEEE Trans. Commun., vol. COM-30, pp. 975-984, May 1982.

[3] E. A. Geraniotis, "Performance of noncoherent direct-sequence spreadspectrum multiple-access communications," IEEE J. Select. Areas Commun., vol. SAC-3, pp. 687-694, Sept. 1985.

[4] J. S. Lehnert and M. B. Pursley, "Error probabilities for binary directsequence spread spectrum communications with random signature sequences," IEEE Trans. Commun., vol. COM-35, pp. 87-98, Jan. 1987.

[5] B. Aazhang and H. V. Poor, "Performance of DS/SSMA communications in impulse channels-Part I: Linear correlation receivers," IEEE Trans. Commun., vol. COM-35, pp. 1179-1188, Nov. 1987.

[6] G. L. Stuber and C. K. Chao, "Analysis of a multiple cell direct sequence CDMA cellular mobile radio system," IEEE J. Select. Areas Commun., vol. 10, pp. 669-679, May 1992.

[7] J. Wang, M. Moeneclay, and L. B. Milstein, "DS-CDMA with predetection diversity for indoor radio communications," IEEE Trans. Commun., vol. 42, pp. 1929-1938, Apr. 1994.

[8] L. B. Milstein, "Interference rejection techniques in spread spectrum communications," Proc. IEEE, vol. 76, pp. 657-671, June 1988.

[9] E. Masry and L. B. Milstein, "Performance of DS spread-spectrum receiver employing interference-suppression filters under a worst-case jamming condition," IEEE Trans. Commun., vol. COM-34, pp. 13-21, Jan. 1986.

[10] L. A. Rusch and H. V. Poor, "Narrowband interference suppression in CDMA spread spectrum communications," IEEE Trans. Commun., vol. 42, pp. 1969-1979, Apr. 1994.

[11] R. A. Iltis and L. B. Milstein, "Performance analysis of narrow-band interference rejection techniques in DS spread spectrum systems," IEEE Trans. Commun., vol. COM-32, pp. 1169-1177, Nov. 1984.

[12] J. W. Ketchum and J. G. Proakis, "Adaptive algorithms for estimating and suppressing narrow-band interference in PN spread spectrum systems," IEEE Trans. Commun., vol. COM-30, pp. 913-923, May 1982.

[13] N. J. Bershad, "Error probabilities for DS spread-spectrum systems using an ALE for narrowband interference rejection," IEEE Trans. Commun., vol. COM-36, pp. 588-595, May 1988.

[14] R. L. Pickholtz, L. B. Milstein, and D. L. Schilling, "Spread spectrum for mobile communications," IEEE Trans. Veh. Technol., vol. 42, pp. 313-322, May 1991.

[15] J. G. Proakis, Digital Communications. New York: McGraw-Hill, 1983.

[16] D. A. S. Fraser, Nonparametric Methods in Statistics. New York: Wiley, 1957.

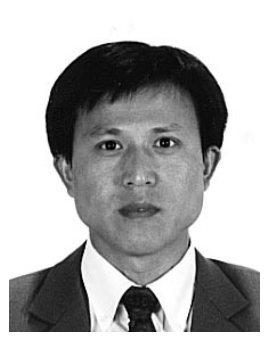

Jiangzhou Wang (M'91-SM'94) received the B.S. and M.S. degrees from Xidian University, Xian, China, in 1983 and 1985, respectively, and the Ph.D degree (with Greatest Distinction) from the University of Ghent, Belgium, in 1990, all in electrical engineering.

From 1990 to 1992 , he was a Post-Doctoral Fellow at the University of California at San Diego, where he worked on the research and development of cellular CDMA systems. From 1992 to 1995, he was a Senior System Engineer at Rockwell International Corporation, Newport Beach, CA, where he worked on the development and system design of wireless communications. Since 1995, he has been with the University of Hong Kong, Hong Kong, where he is currently an Associate Professor. He is teaching and conducting research in the areas of cellular mobile and spread-spectrum communications. He holds one U.S. patent in the GSM system.

Dr. Wang has been a committee member of a number of international conferences. He is an Editor for the IEEE TRANSACTIONS ON COMMUNICATIONS and a Guest Editor for the IEEE JouRnAl ON SELECTED AREAS IN COMMUNICATIONS. 